\title{
Half-Sine and Triangular Despreading Chip Waveforms for Coherent Delay-Locked Tracking in DS/SS Systems
}

\author{
Subramaniam Thayaparan, Tung-Sang Ng, Senior Member, IEEE, and Jiangzhou Wang, Senior Member, IEEE
}

\begin{abstract}
The performance of a coherent delay-locked tracking scheme for direct-sequence/spread-spectrum systems using half-sine or triangular chip waveforms for early and late despreading sequences is analyzed. The effect of band-limiting on the received signals is considered. Mean time to lose lock (MTLL) and root mean square (rms) tracking error of the delay-locked loop (DLL) are compared with that of a conventional DLL which uses rectangular chip despreading waveforms. Linear and nonlinear (based on the renewal process approach) analyses are employed to evaluate the performance of the DLL. Results show that the use of either the half-sine or triangular chip waveform reduces the rms tracking error and increases the MTLL considerably when the early-late spacing is approximately between $0.7-1.3$ chip times. The results apply in particular to the commonly used DLL using one chip early-late spacing. Computer simulation for band-limited signals confirms the analytical results. The use of these despreading chip waveforms also reduces tracking offset in multipath environments.
\end{abstract}

Index Terms-Chip waveforms, delay-locked loop, direct-sequence spread spectrum, PN code tracking, synchronization.

\section{INTRODUCTION}

$\mathbf{C}$ OMMERCIAL applications of direct-sequence/spreadspectrum (DS/SS) systems have increased substantially in recent years, especially in mobile communications and positioning systems. Such systems require that the received and the locally generated spreading waveforms to be synchronized. The delay-locked loop technique is widely used for tracking purposes. To ensure low bit-error rates (BER's) during normal operation, small tracking error in the delay-locked loop (DLL) should be maintained. Even though mean time to lose lock (MTLL) is much larger than the average connection time under normal circumstances, sporadic signal fading during transmission may cause sudden reduction in the received signal-to-noise ratio (SNR) and the tracking loop to lose lock. The MTLL is therefore an important parameter used to measure system performance. To ensure good system performance, the tracking

Paper approved by C. Robertson, the Editor for Spread Spectrum Systems of the IEEE Communications Society. Manuscript received July 6, 1998; revised January 12, 1999 and September 1, 1999. This work was supported by the Hong Kong Research Grants Council (RGC) of The Hong Kong Government and the CRCG of The University of Hong Kong.

The authors are with the Department of Electrical and Electronic Engineering, The University of Hong Kong, Hong Kong (e-mail: sthay @eee.hku.hk; tsng@eee.hku.hk; jwang@eee.hku.hk).

Publisher Item Identifier S 0090-6778(00)07097-5. error and MTLL should be kept as small and large, respectively, as possible [1]-[3]. Multipath signals introduce a tracking offset in the DLL and an additional SNR may be required to achieve the desired BER. Therefore, the tracking offset of the DLL should also be kept as small as possible [4]-[6].

The performance of the DLL has been analyzed in many papers. Substantial work has been done in the past to enhance the performance by modifying the DLL structure, for example, [7]-[10]. However most of these modifications focus on the structure of the DLL, which changes the S-curve, for example, [9]. The S-curve of the DLL is highly dependent on the locally generated chip waveform since it is obtained as the difference of the early and late correlation functions. These papers analyze DLL performance by considering the normalized (chip time) cross correlation function $R(\tau)$ of the incoming and locally generated spreading codes as a triangular function, where $R(\tau)=1-|\tau|$ for $|\tau| \leq 1$ and $R(\tau)=0$, otherwise. In practice, this happens only if the received and locally generated chip waveforms are rectangular in shape.

The employment of nonrectangular spreading chip waveforms for DS/CDMA transmitted signals has been discussed in [11] and [12]. Recently, weighted despreading chip waveforms, while the spreading chip waveform is the conventional rectangular, have been proposed [13]-[16] for DS/CDMA systems. The purpose is to optimize the shape of the chip waveforms in the receiver to reduce the multiple-access interference (MAI) and to increase capacity. In this paper, the effect of nonrectangular despreading chip waveforms for locally generated early and late despreading codes in a coherent DLL for DS/SS systems is investigated. The transmitted signal is assumed to be conventional with a rectangular spreading chip waveform. The waveforms considered include half-sine and triangular, and are assumed to be time limited to the chip duration which causes no interchip interference. The code tracking loop considered here is a first order DLL with an arbitrary early-late spacing.

The rest of the paper is organized as follows. In Section II, the system model is described. Section III presents the performance analysis when the received signal is not band-limited (ideal) and corrupted by the additive white noise. Section IV investigates the effect of band-limiting of the received signal on the performance of the DLL. Numerical examples are given in Section V. The effect of multipath signals is considered in Section VI. Finally, discussion, and conclusions are given in Sections VII and VIII, respectively. 


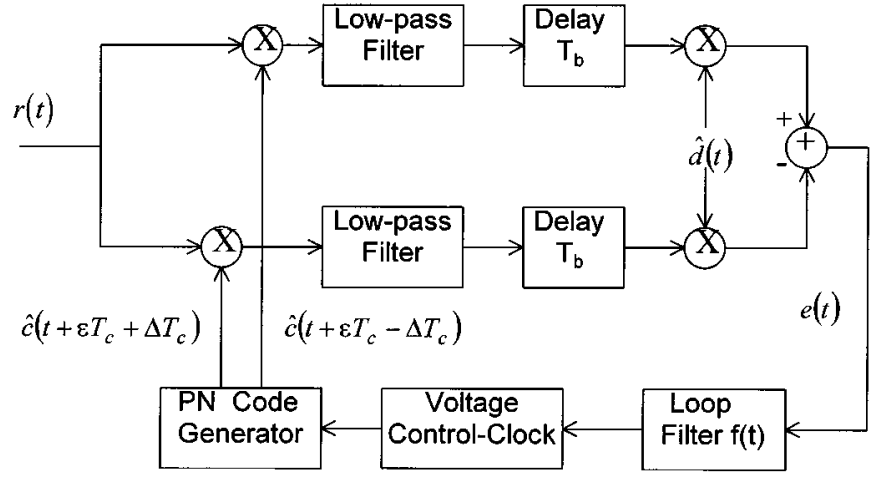

Fig. 1. Block diagram of the coherent DLL in baseband.

\section{SYSTEM MODEL}

In the following, a coherent DLL is considered and signals are analyzed in the baseband. The block diagram of a DLL in the baseband is given in Fig. 1. The baseband received signal $r(t)$ in a DS/SS system can be expressed as

$$
r(t)=\sqrt{P} d(t) c(t) \cos (\eta)+n(t)
$$

where $P$ is the received signal power, $d(t)$ is a binary data stream, $c(t)$ is the data-synchronous PN code sequence, $\eta$ is the phase difference between the locally generated and the incoming carrier signals, and $n(t)$ is the additive white noise with double-sided power spectral density of $N_{o} / 2$. The spreading modulation is assumed to be BPSK (binary phase-shift keying) with conventional rectangular chip spreading waveform. The early-late spacing of the DLL is denoted by $2 \Delta T_{c}$ where the parameter $\Delta(0<\Delta \leq 1)$ is an arbitrary value and $T_{c}$ is the chip time. Thus, the early and late despreading sequences of the DLL are separated by $\Delta T_{c}$ from the despreading sequence which is used for data demodulation.

In the conventional DLL, the early and late despreading sequences are obtained as shifted versions of the locally generated $c(t)$, denoted by $\hat{c}(t)$. Conventionally, the chip waveform of $\hat{c}(t)$ is $p(t)$ where $p(t)=1$ for $0 \leq t<T_{c}$ and $p(t)=0$, otherwise. Now consider i) a half sinusoidal pulse $\hat{p}_{s}(t)$ and ii) a triangular pulse $\hat{p}_{t}(t)$, instead of the commonly used rectangular pulse $p(t)$. Fig. 2 shows the chip arrangements of single early and late PN pulses with an incoming PN pulse for $\Delta=1 / 2$ when the received and the locally generated pseudonoise $(\mathrm{PN})$ sequences are aligned with negligible phase error. We use the subscript $s$ and $t$ to denote the corresponding parameter of half-sine and triangular chip waveforms, respectively, i.e.,

$$
\hat{p}_{s}(t)= \begin{cases}\sin \left(\frac{\pi t}{T_{c}}\right), & 0 \leq t<T_{c} \\ 0, & \text { otherwise }\end{cases}
$$

and

$$
\hat{p}_{t}(t)= \begin{cases}1-\left|\frac{2 t}{T_{c}}-1\right|, & 0 \leq t<T_{c} \\ 0, & \text { otherwise }\end{cases}
$$
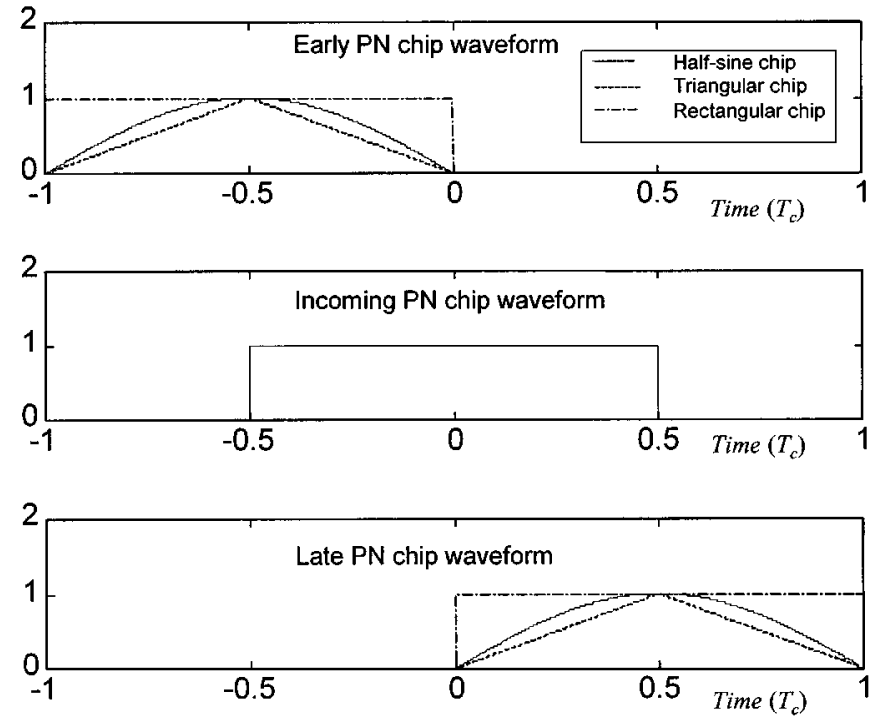

Fig. 2. Early, late, and incoming PN pulses.

For the triangular chip waveform case, the maximum value of $\hat{p}_{t}(t)$ is fixed at the middle $\left(t=T_{c} / 2\right)$ of the chip waveform. Otherwise, the early and late despreading signals require two different chip waveforms, which are mirror images to each other, and the reason will be given in Section III-A. Since the chip waveforms are restricted to the duration $T_{c}$, there is no interchip interference. These chip waveforms $\hat{p}_{s}(t)$ and $\hat{p}_{t}(t)$ are normalized by limiting their maximum value to 1 . We assume that the interval of each data bit in $d(t)$ is $T_{b}$ and is equal to the period of the despreading sequence $N T_{c}$ where $N$ is the PN sequence length.

\section{PERformance AnAlysis}

The effect of the data signal $d(t)$ in the coherent code tracking loop is removed by multiplying (decision-feedback) the early and late signals with the estimated binary data stream $\hat{d}(t)$ as shown in Fig. 1. The correlated signals at the early and late branches of the DLL are delayed by a data-bit time $T_{b}$ in order to cancel the delay introduced by $\hat{d}(t)$ due to the demodulation process. A similar technique, which cancels the effect of data signal in a coherent DLL, can be found in [17]. Under perfect conditions, the average value of $d(t) \hat{d}(t)$ denoted by $\overline{d(t) \hat{d}(t)}$ is 1 . Under practical situations, the value of $\overline{d(t) \hat{d}(t)}$ will be less than 1 due to both the estimation error in $\hat{d}(t)$ and the effects of filtering. It is clear from (1) that the parameters $d(t) \hat{d}(t)$ and $\cos (\eta)$ can be grouped with the received signal power $P$. Note that both terms $\overline{d(t) \hat{d}(t)} \leq 1$ and $\cos (\eta) \leq 1$. Therefore, the term $\sqrt{P}\{\overline{d(t) \hat{d}(t)}\} \cos (\eta)$ can be considered as a degradation on the received signal power $P$. This degradation is independent of the local despreading chip waveform used in the tracking loop. Thus, the performance degradation of the coherent DLL due to $\overline{d(t) \hat{d}(t)}$ and $\cos (\eta)$ can be separately expressed in terms of the reduction in SNR. From this point onwards, we shall consider the resultant received signal power $P_{o}=P\{\overline{d(t) \hat{d}(t)}\}^{2} \cos ^{2}(\eta)$. 


\section{A. Stochastic Differential Equation}

The cross correlation function $R(\tau)$ of the incoming and the locally generated PN sequences is given by

$$
R(\tau) \triangleq \frac{1}{N T_{c}} \int_{0}^{N T_{c}} c(t) \hat{c}\left(t+\tau T_{c}\right) d t .
$$

We assume that the PN sequence is very long so that $R(\tau)$ vanishes when $|\tau| \geq 1$. Combining (2) and (3) with (4), the respective cross correlation functions are obtained as

$$
R_{s}(\tau)= \begin{cases}\frac{1}{\pi}\{1+\cos (\pi \tau)\}, & |\tau|<1 \\ 0, & \text { otherwise }\end{cases}
$$

and

$$
R_{t}(\tau)= \begin{cases}\frac{1}{2}-|\tau|^{2}, & |\tau|<\frac{1}{2} \\ (1-|\tau|)^{2}, & \frac{1}{2} \leq \tau<1 \\ 0, & \text { otherwise }\end{cases}
$$

The cross correlation functions $R_{s}(\tau)$ and $R_{t}(\tau)$ are symmetric due to the symmetric chip waveforms $\hat{p}_{s}(t)$ and $\hat{p}_{t}(t)$. If the triangular chip waveform $\hat{p}_{t}(t)$ is not symmetric, the cross correlation function $R_{t}(\tau)$ will not be symmetric and it follows that the discriminator characteristic function of the DLL, generated by the shifted versions of $R_{t}(\tau)$ used as early and late cross correlation functions, will introduce a tracking offset. Alternatively, we can use two different chip waveforms $\hat{p}_{t+}(t)$ and $\hat{p}_{t-}(t)$, which are mirror images of each other, for the early and late despreading sequences. We also observe from (5) and (6) that the slope of $R(\tau)$ is maximum for both triangular and half sine chip waveforms at $\tau= \pm 0.5$.

The delay-locked discriminator output in the absence of white noise is obtained by the difference of the early and late cross correlation functions and it is given by $\sqrt{P_{o}} S(\varepsilon)$, where $S(\varepsilon)$ is the discriminator S-curve [3] and $\varepsilon$ is the phase error normalized to $T_{c}$.

$$
S(\varepsilon)=R(\varepsilon-\Delta)-R(\varepsilon+\Delta) .
$$

Let $S^{\prime}(\varepsilon)=d S(\varepsilon) / d \varepsilon$. The slope at the origin given by $S^{\prime}(0)$ plays a major role in the performance of the DLL as we shall see in Section III-B. Tracking performance is improved with increasing $S^{\prime}(0)$. From (7), the slope $S^{\prime}(0)$ can be expressed as $R^{\prime}(-\Delta)-R^{\prime}(+\Delta)$ where $R^{\prime}(\tau)=d R(\tau) / d \tau$. It is now clear from (5) and (6) that the maximum values of the chip waveforms, $\hat{p}_{s}(t)$ and $\hat{p}_{t}(t)$, in (2) and (3) at $t=T_{c} / 2$ achieves the maximum slope $S^{\prime}(0)$ for the early-late spacing $\Delta=1 / 2$. The plots of the $S$-curves $S(\varepsilon)$ for the three chip waveforms, rectangular, half sine, and triangular, are presented in Fig. 3 for $\Delta=1 / 2$. We note that the slope of $R(\tau)$ at $\tau= \pm 1 / 2$ is the same for all three chip waveforms, as is that of $S(\varepsilon)$ at $\varepsilon=0$. We also observe that the linear regions of the $\mathrm{S}$-curves for half-sine and triangular chip waveforms have substantially reduced compared to that of rectangular pulses. Therefore, a change in threshold values, where the results of linear and nonlinear analyses differs, can be expected. This also makes the use of nonlinear analysis to evaluate the DLL performances more

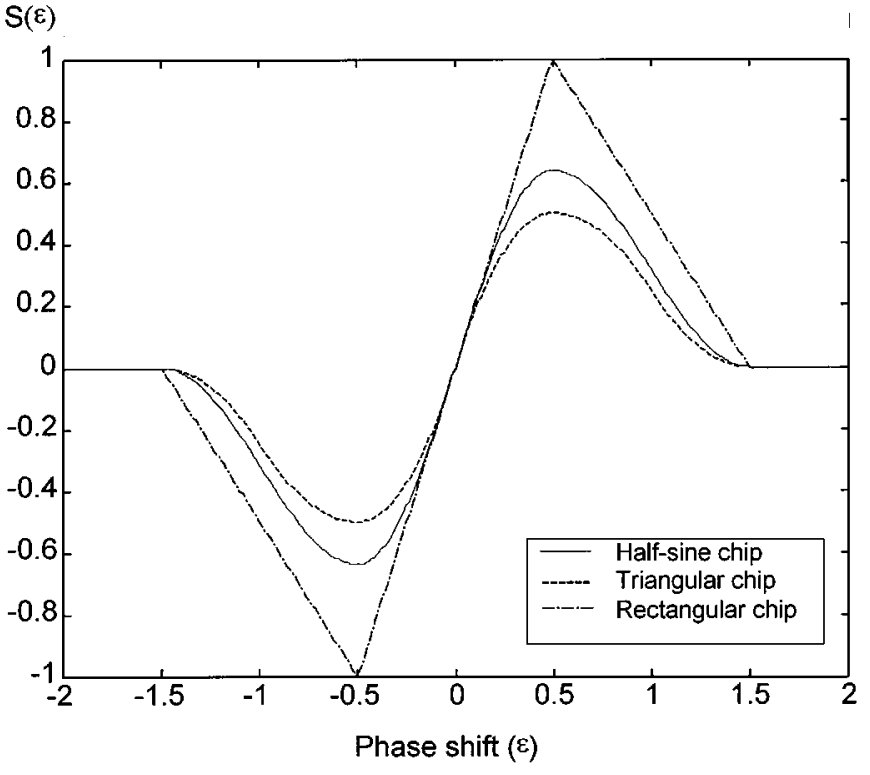

Fig. 3. S-curves for the half-sine, triangular, and rectangular chip waveforms.

appropriate. Referring to Fig. 1, the error signal $e(t)$ can be expressed as

$$
e(t)=\sqrt{P_{o}} S(\varepsilon)+n_{T}(t)
$$

where the total noise $n_{T}(t)$ is given by

$$
n_{T}(t)=\hat{d}(t)\left\{\hat{c}\left(t+\varepsilon T_{c}-\Delta T_{c}\right)-\hat{c}\left(t+\varepsilon T_{c}+\Delta T_{c}\right)\right\} n(t) .
$$

As shown in Fig. 1, the error signal at the output of the loop filter drives the voltage controlled oscillator (VCO), shown as $\mathrm{PN}$ code generator, to correct the code phase error. The operation of the VCO is described by [3]

$$
\varepsilon(t)=-K \int_{0}^{t} f(z) \otimes e(z) d z
$$

where $f(t)$ is the impulse response of the loop filter, $K$ is the VCO sensitivity gain, and $\otimes$ denotes convolution. Combining (8) and (10), the stochastic differential equation that describes the dynamic behavior of the tracking loop is obtained

$$
\frac{d \varepsilon}{d t}=-K\left\{\sqrt{P_{o}} S(\varepsilon)+n_{T}(t)\right\} \otimes f(t) .
$$

In deriving (11), we assumed that the channel is slowly time varying such that the Doppler spread can be neglected. A firstorder code tracking loop is considered in the following analysis and therefore $f(t)=\delta(t)$ where $\delta(t)$ is a unit impulse function.

\section{B. Linear Analysis}

The root-mean-square (rms) tracking error for the DLL can be obtained by linear loop analysis and details of this analysis for conventional system can be found in [3, Ch. 4]. The results so obtained are generally considered as good approximations to the actual performance in the region of SNR which we are interested in. The high SNR assumption will result in smaller tracking error for the DLL and this enables us to approximate $S(\varepsilon)$ as linear in the vicinity of $\varepsilon \approx 0$. Thus, $S(\varepsilon)$ is substituted 
by $S^{\prime}(0) \varepsilon$ for all three chip waveforms and (11) can be rewritten for small tracking errors as

$$
\frac{d \varepsilon}{d t}=-K\left\{\sqrt{P_{o}} S^{\prime}(0) \varepsilon+n_{T}(t)\right\} .
$$

The autocorrelation function of the white noise $n(t)$ is an impulse function, and so is the autocorrelation function of the total noise process $n_{T}(t)$ [3]. Note that the noise process $n_{T}(t)$ is white but not Gaussian, as shown by

$$
\begin{aligned}
R_{n_{T}}(\tau) & =E\left\{n_{T}(t) n_{T}\left(t+\tau T_{c}\right)\right\} \\
& =\left\{R_{\hat{c}}(0)-R_{\hat{c}}(2 \Delta)\right\} N_{0} \delta(\tau)
\end{aligned}
$$

where $R_{\hat{c}}(\tau)$ is the normalized autocorrelation function of locally generated PN code $\hat{c}(t)$. In deriving (13), the expectation $E\left\{\hat{d}^{2}(t)\right\}=1$ is used. Denote the power spectral density of the total noise process $n_{T}(t)$ by $S_{n_{T}}(f)$. The Fourier transform of $R_{n_{T}}(\tau)$ gives $S_{n_{T}}(f)$, which is flat with a spectral level of $\left\{R_{\hat{C}}(0)-R_{\hat{C}}(2 \Delta)\right\} N_{0}$. The closed-loop transfer function of the first-order DLL is

$$
H(s)=\frac{\sqrt{P_{o}} S^{\prime}(0) K}{s+\sqrt{P_{o}} S^{\prime}(0) K} .
$$

The closed-loop transfer function and the closed-loop bandwidth depend on the early-late spacing for all three chip waveforms due to the change in $S^{\prime}(0)$. The closed-loop bandwidth of the DLL is given by

$$
W_{L}=\int_{0}^{\infty}|H(j 2 \pi f)|^{2} d f=\frac{\sqrt{P_{o}} S^{\prime}(0) K}{4} .
$$

Thus, the root mean square (rms) tracking error of the DLL, $\sigma$, can be obtained using linear loop analysis for ideal received signals as

$$
\sigma=\sqrt{\frac{2\left\{R_{\hat{c}}(0)-R_{\hat{c}}(2 \Delta)\right\}}{\gamma_{d} S^{\prime}(0)^{2}}}
$$

where $\gamma_{d}=P_{o} / N_{o} W_{L}$ is the effective loop SNR. We note that the linear analysis is valid only under normal operating conditions where the received SNR is high enough. Under these circumstances the MTLL is not of much interest since it is much longer than the transmission time.

\section{Nonlinear Analysis}

Nonlinear analysis is found useful when the system operates at threshold or below. In these situations, the MTLL is of primary consideration. The MTLL is defined by the time taken for the timing error $\varepsilon$ to reach one of its boundaries either $\varepsilon=\varepsilon_{\max }$ or $\varepsilon=\varepsilon_{\min }$ from the stable equilibrium point $\varepsilon=0$. Therefore, the range of the S-curve becomes $\varepsilon_{\max }$ to $\varepsilon_{\min }$. The average behavior of the system is characterized by the stationary probability density function (pdf) $p(\varepsilon)$ of the phase error $\varepsilon$. We follow the analysis given in [18] and [19] in which an aperiodic S-curve of the DLL determines $p(\varepsilon)$. Under practical situations where the bandwidth of the noise process $n_{T}(t)$ is much greater than the closed-loop bandwidth $W_{L}$, the function $G(\varepsilon)$ satisfies the following Fokker-Plank equation [18]-[20]:

$$
\frac{\partial}{\partial \varepsilon}\left\{K_{1}(\varepsilon) G(\varepsilon)\right\}-\frac{1}{2} \frac{\partial^{2}}{\partial \varepsilon^{2}}\left\{K_{2}(\varepsilon) G(\varepsilon)\right\}=\pi(\varepsilon)
$$

where $\pi(\varepsilon)$ is the initial pdf of the phase error $\varepsilon$ when the acquisition unit hands over to the tracking unit, and the parameters $K_{1}(\varepsilon)$ and $K_{2}(\varepsilon)$ are defined by

$$
\begin{aligned}
& K_{1}(\varepsilon)=-K \sqrt{P_{o}} S(\varepsilon) \\
& K_{2}(\varepsilon)=K^{2} \int_{-\infty}^{+\infty} R_{n_{T}}(\delta) d \delta=K^{2} S_{n_{T}}(0) .
\end{aligned}
$$

In (18) and (19), $S(\varepsilon)$ and $S_{n_{T}}(0)$ depend on the chip waveform, and hence $K_{1}(\varepsilon)$ and $K_{2}(\varepsilon)$ depend on the choice of chip waveform. The in-lock region of the loop is denoted by $\Re \triangleq\left(\varepsilon_{\min }, \varepsilon_{\max }\right)$ in which the acquisition unit hands over to the tracking unit for fine synchronization. When the SNR is low, the lock detector indicates the true lock condition when sufficient code correlation is present. This happens near the zero offset position and with very low probability elsewhere. Therefore, a suitable expression for $\pi(\varepsilon)$ is a delta function [18, p. 33]. Furthermore, according to the MTLL definition, the first passage of $\varepsilon$ starts from $\varepsilon=0$ and, therefore, the solution of the differential equation in (17) with $\pi(\varepsilon)=\delta(\varepsilon)$ can be used to evaluate both rms tracking error and MTLL. Assuming $\pi(\varepsilon)=\delta(\varepsilon)$, the following expression for $G(\varepsilon)$ is obtained [18]:

$$
G(\varepsilon)=\frac{e^{-U(\varepsilon)}}{K_{2}(\varepsilon)} \int_{\varepsilon}^{\varepsilon_{\max }} e^{U(z)} d z, \quad 0 \leq \varepsilon \leq \varepsilon_{\max }
$$

where

$$
U(\varepsilon)=-2 \int_{\varepsilon_{\min }}^{\varepsilon} \frac{K_{1}(z)}{K_{2}(\varepsilon)} d z .
$$

Note that $G(\varepsilon)$ is an even function [18], [20]. The probability density function $p(\varepsilon)$ is related to the MTLL, which is denoted by $\bar{T}$, and $G(\varepsilon)$ by

$$
p(\varepsilon)=\left\{1-P_{L D}(\varepsilon)\right\} \frac{G(\varepsilon)}{\bar{T}}, \quad \varepsilon_{\min } \leq \varepsilon \leq \varepsilon_{\max }
$$

where $P_{L D}(\varepsilon)$ is the probability that the tracking unit declares out of lock when $\varepsilon$ is in the region of $\Re$ and it is assumed that $P_{L D}\left(\varepsilon_{\min }\right)=P_{L D}\left(\varepsilon_{\max }\right)=1$, i.e., the boundaries are absorbing. The following expressions for MTLL $(\bar{T})$ and rms tracking error $(\sigma)$ are derived using (20) and (22):

$$
\bar{T}=\int_{\varepsilon_{\min }}^{\varepsilon_{\max }}\left\{1-P_{L D}(\varepsilon)\right\} G(\varepsilon) d \varepsilon
$$

and

$$
\sigma^{2}=\int_{\varepsilon_{\min }}^{\varepsilon_{\max }} \varepsilon^{2} p(\varepsilon) d \varepsilon .
$$

By assuming a suitable function for $P_{L D}(\varepsilon)$, the solutions of (21), (20), (23), (22), and (24) are obtained in the respective order by using numerical integration. 


\section{BAND-LiMited RECEIVED SignaL}

In practice, all received signals are band-limited. Generally, the transmission channel consists of a transmitter filter, a receiver filter, and a propagation medium. The equivalent transfer functions of these are denoted by $H_{T}(f), H_{R}(f)$, and $H_{M}(f)$, respectively. As a result, the equivalent low-pass transfer function of the transmission channel $H_{C}(f)$ becomes

$$
H_{C}(f)=H_{T}(f) H_{M}(f) H_{R}(f) .
$$

Note that the transfer function of the propagation medium depends on the channel environment. We assume that the propagation medium is ideal and therefore $H_{M}(f)=1$. This assumption can be justified since our intention is to study the effect of band-limiting on the received signal. The impulse response of the transmission channel is denoted by $h_{c}(t)$ and this is the inverse Fourier transform of $H_{C}(f)$. In practice, due to the influence of $H_{C}(f)$, the received chip waveform $\bar{p}(t)$ will not be limited in the range of $0 \leq t<T_{c}$. As a result, the expression for the cross correlation $R(\tau)$ given in (4), which is still valid, is not limited to $|\tau|<1$. The band-limited received signals are modeled by assuming an ideal low-pass transfer function for $H_{C}(f)$. Even though this is a rough approximation for $H_{C}(f)$, it serves the purpose of showing the effects of band-limiting on the received signals. For illustration purposes, assume

$$
H_{C}(f)= \begin{cases}1, & |f| \leq \frac{2}{T_{C}} \\ 0, & \text { otherwise }\end{cases}
$$

i.e., the bandwidth of the channel is assumed to be twice that of the main lobe spectrum of the transmitted PN signal. Using the expression for $h_{c}(t)$ of (26), the received PN signal $c(t) \otimes$ $h_{c}(t)$, denoted by $\bar{c}(t)$, has the chip waveform $\bar{p}(t)$ where $\bar{p}(t)=$ $p(t) \otimes h_{c}(t)$. Replacing $\bar{p}(t)$ for $p(t), R(\tau)$ in (4) for bandlimited received signals is obtained.

In the case of linear analysis, the band-limiting of the received signal affects the parameter $S^{\prime}(0)$ in the rms tracking error expression given in (16). The parameter $S^{\prime}(0)$ is computed using numerical integration. For the nonlinear analysis, $S(\varepsilon)$ in (7) has to be obtained using $\bar{c}(t)$. Thus, replacing new $K_{1}(\varepsilon)$ in (18), the MTLL and the tracking error in (23) and (24) are computed using numerical integration. We note that the change in $S^{\prime}(0)$ due to band-limiting will also cause variation in $H(j 2 \pi f)$ in (14) and $W_{L}$ in (15) for both linear and nonlinear analyses. In practice, $H(j 2 \pi f)$ and $W_{L}$ can be adjusted by varying the sensitivity gain $K$ of the voltage-controlled clock in the DLL.

\section{NUMERICAL EXAMPLE}

When the received signal is ideal, the parameter $S^{\prime}(0)$, which is given by $R^{\prime}(-\Delta)-R^{\prime}(+\Delta)$, can be obtained by differentiating the cross correlation functions $R_{s}(\tau)$ in (5) and $R_{t}(\tau)$ in (6) with respect to the phase shift $\tau$. For band-limited received signals, the values of $S^{\prime}(0)$ are obtained by numerical integration for all chip waveforms. The autocorrelation values $R_{\hat{c}}(\tau)$ for rectangular and half-sine waveforms are, respectively, $1-\tau$ and $\{\pi(1-\tau) \cos (\pi \tau)+\sin (\pi \tau)\} / 2 \pi$ for $\tau \leq 1$. The values of $R_{\hat{c}}(\tau)$ for triangular chip waveform is $1 / 3-2 \tau^{2}+2 \tau^{3}$ for

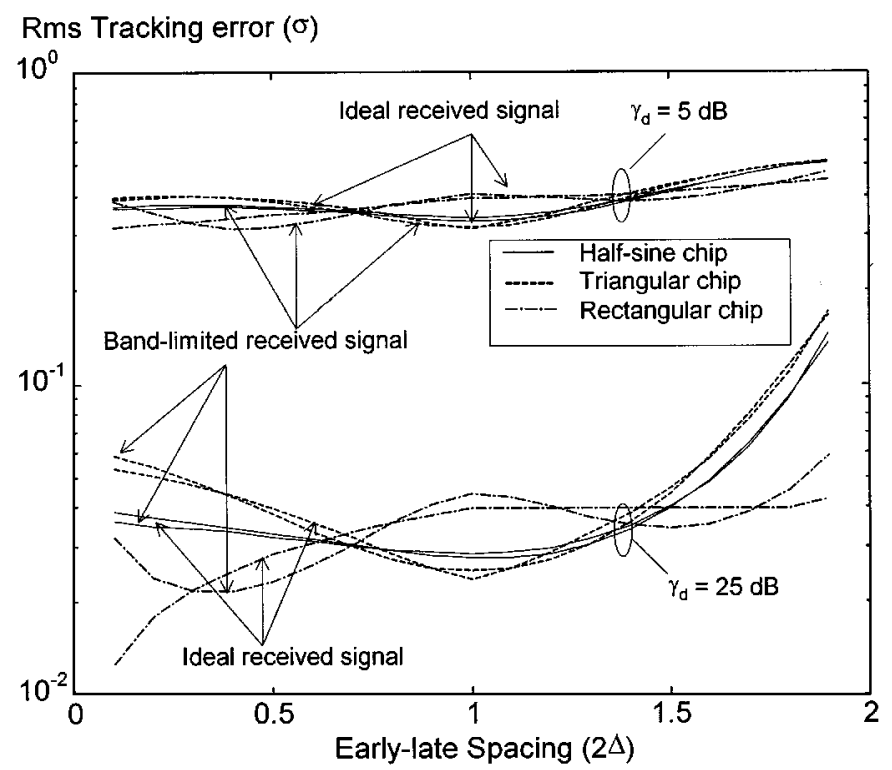

Fig. 4. RMS tracking error against the early-late spring.

$\tau \leq 1 / 2$ and $2(1-\tau)^{3} / 3$ for $1 / 2<\tau \leq 1$. The value of $R_{\hat{c}}(\tau)$ is zero for all chip waveforms when $\tau>1$.

Following the procedures explained in Section III-C, the MTLL and the rms tracking errors are obtained for all three chip waveforms using the nonlinear analysis with the assumption

$$
P_{L D}(\varepsilon)= \begin{cases}0, & |\varepsilon|<1 \\ 1, & \text { otherwise }\end{cases}
$$

When the phase error $\varepsilon$ of the incoming and locally generated PN sequence exceeds 1 , the data demodulation can not be performed since the cross correlation value $R(\tau)$ in (4) vanishes. The lock detector therefore may declare the loop out of lock. Thus, the assumption made in (27) is clearly justifiable when the lock detector has no faulty triggering.

\section{A. Early-Late Spacing}

The effect of the early-late spacing $(2 \Delta)$ on the performance of the DLL is first investigated. Due to the wide range of $\Delta$ being considered, nonlinear analysis yields more accurate results and is therefore used. Fig. 4 plots the rms tracking error against the early-late spacing for the effective loop SNR $\gamma_{d}$ equal to 5 and $25 \mathrm{~dB}$. Fig. 5 shows the MTLL against the early-late spacing for $\gamma_{d}$ equal to 5 and $10 \mathrm{~dB}$. First, these graphs indicate that within the range of $0.7-1.3$ chip times, the half-sine and triangular chip waveforms reduce tracking error and increase MTLL. Second, band-limiting has little effect on tracking performance for all three waveforms. Third, the conventional rectangular despreading waveform has better tracking performance when $2 \Delta$ is less than 0.7 ; however, band-limiting severely degrades its performances as $\Delta$ decreases. From the acquisition point of view, one chip early-late spacing is preferred. It is also clear from Figs. 4 and 5 that the performance of the DLL with one chip early-late spacing would be improved by using triangular and half-sine chip waveforms. In the following section, we shall evaluate the performance of the DLL with one chip early-late spacing $(\Delta=1 / 2)$. 


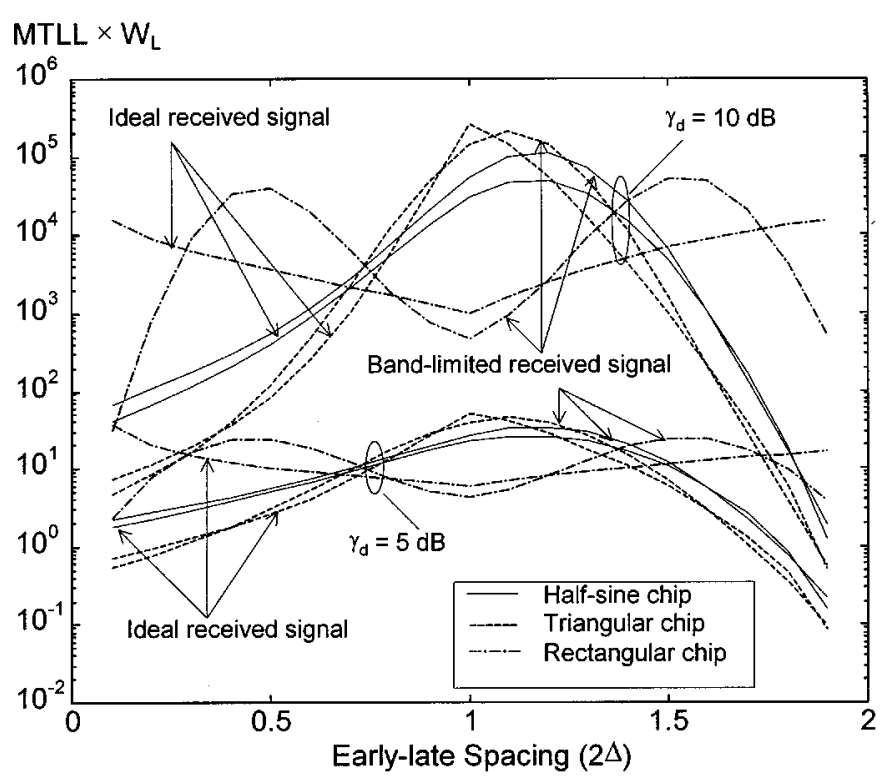

Fig. 5. MTLL against the early-late spacing.

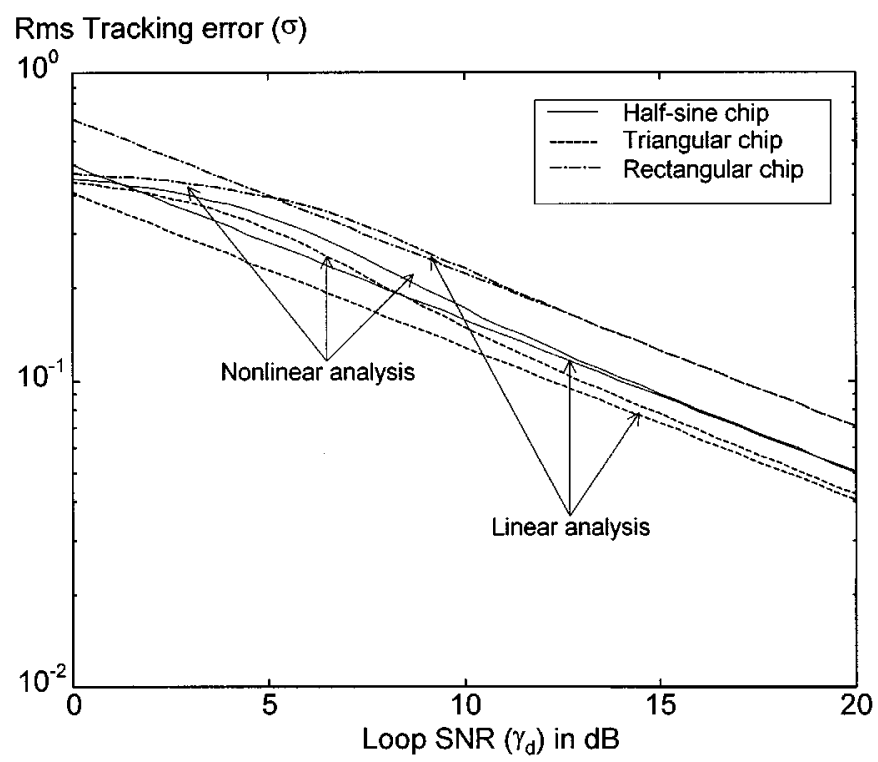

Fig. 6. RMS tracking error against loop SNR for ideal received signal.

\section{B. DLL with One Chip Time Early-Late Spacing}

The rms tracking error of the DLL, obtained by both linear and nonlinear analysis, against the loop SNR $\left(\gamma_{d}\right)$ is plotted for the three cases in Fig. 6 for ideal received signal and in Fig. 7 for band-limited received signals. These results indicate good agreement between linear and nonlinear analysis under the conditions considered. The triangular chip waveform has the smallest tracking error among the three chip waveforms. Both ideal and band-limited signals give the same conclusions. It is clear from Fig. 6 that for a specific tracking error, triangular and half-sine chip waveforms reduce the required loop SNR by approximately 4.77 and $3 \mathrm{~dB}$, respectively. However, from Fig. 7, band-limiting of the received signal requires an increase of loop SNR by approximately $1.2 \mathrm{~dB}$ for the rectangular and $0.9 \mathrm{~dB}$ for the triangular chip waveforms. The rms tracking error for
Rms Tracking error $(\sigma)$

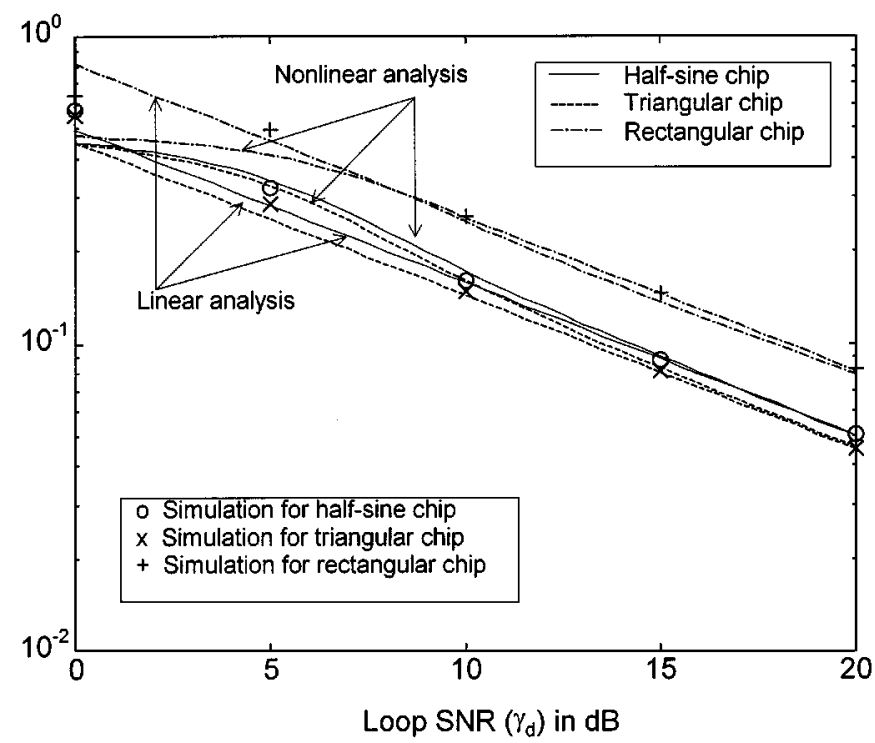

Fig. 7. RMS tracking error against loop SNR for band-limited signals.

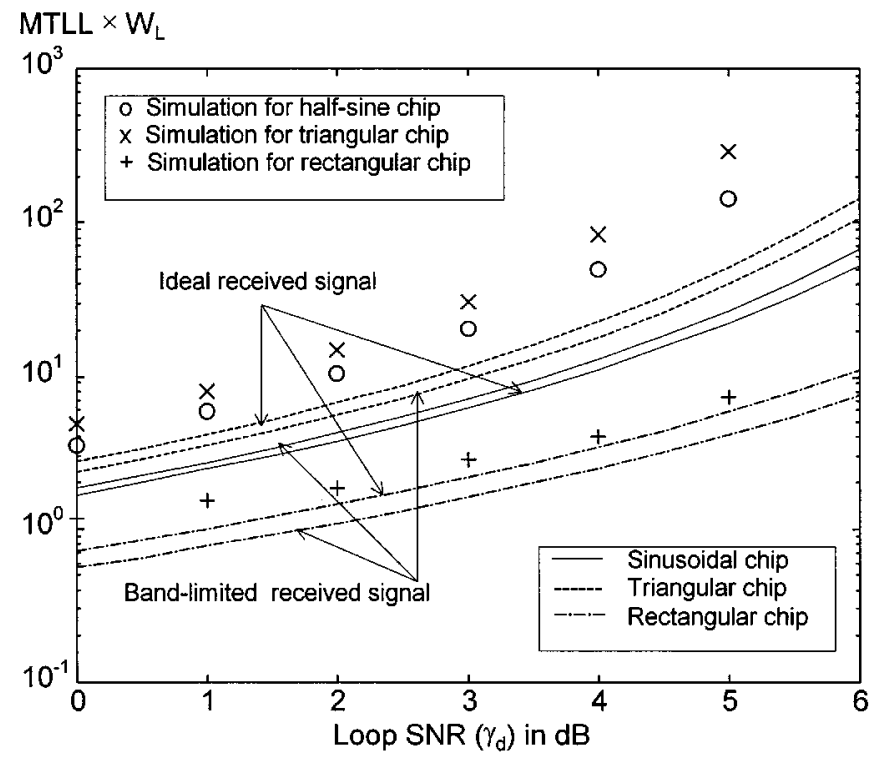

Fig. 8. Plots of MTLL against loop SNR.

the half-sine chip waveform does not seem to be affected much by band-limiting.

Fig. 8 shows the curves of MTLL for both ideal and band-limited received signals. For a specific loop SNR, the triangular chip waveform has the longest MTLL while the rectangular chip waveform has the lowest MTLL among the three chip waveforms. A band-limited received signal reduces the MTLL as expected. However, the effect of the half-sine chip waveform is minimal.

\section{Simulations}

The DLL scheme using the three different despreading waveforms was simulated by a computer for band-limited received signal using a maximal-length sequence with $N=255$. The rms tracking error is obtained by using 10000 sample points for each $\gamma_{d}$ at $0,5,10,15$, and $20 \mathrm{~dB}$. The results are marked 
in Fig. 7 and they show a close agreement with the results obtained analytically. The simulated results for MTLL using the mean of 1000 times to lose lock for $\gamma_{d}$ at $1 \mathrm{~dB}$ intervals are also marked in Fig. 8. These simulated results for MTLL are higher than those obtained by analytical means. However, the differences between the MTLL by using different chip waveforms are approximately the same as those obtained analytically.

\section{EFFECT OF MULTIPATH SignALS}

It is well known that the DLL will introduce a tracking offset when multipath signals are present [4]. An investigation of the effect of tracking offset when the received signal consists of two rays, one direct and a reflected ray, follows. Band-limiting of the received signals is not considered in this analysis. The received signal in (1) for the two-ray model can be expressed as

$$
\begin{array}{r}
r(t)=\sqrt{P}\left\{d(t) c(t)+\alpha d\left(t-\beta T_{c}\right) c\left(t-\beta T_{c}\right) \cos (\zeta)\right\} \\
\cdot \cos (\eta)+n(t)
\end{array}
$$

where $\alpha$ is the attenuation, $\beta$ is the time delay normalized to $T_{c}$, and $\zeta$ is the carrier phase difference of the reflected ray with respect to the direct ray. Note that the values of $\overline{d(t) \hat{d}(t)}$ and $d\left(t-\beta T_{c}\right) \hat{d}(t)$ are approximately the same since the delay $\beta T_{c}$ is negligible compared with the PN code length $N T_{c}$. Thus, the new $\mathrm{S}$-curve of the DLL $S_{m}(\varepsilon)$ becomes

$$
S_{m}(\varepsilon)=S(\varepsilon)+\alpha S(\varepsilon+\beta) \cos (\zeta) .
$$

The maximum tracking offset occurs when the reflected ray is in phase or out of phase with the specular signal. In this case, the carrier phase estimate will have negligible error since the carrier tracking loop tracks the sum of the specular and diffuse path signals [4]. The tracking offset for any value of phase difference $\zeta$ and delay $\beta$ is the solution of $S_{m}(\varepsilon)=0$. The absolute value of the maximum tracking offset for the worst case of phase difference $\zeta$ and delay $\beta$ is denoted by $\varepsilon_{o}$, and this happens when the second term in (29) has the maximum value for all possible $\zeta$ and $\beta$. Therefore, $\varepsilon_{0}$ is the solution of the equation

$$
S(\varepsilon)-\alpha \times \max |S(\varepsilon)|=0 .
$$

By solving (30), we obtain $\varepsilon_{o}=\alpha / 2$ for the rectangular waveform, $\varepsilon_{o}=\sin ^{-1}(\alpha) / 2$ for the half-sine waveform, and $\varepsilon_{o}=$ $(1-\sqrt{1-\alpha}) / 2$ for the triangular waveform, respectively. The plots of $\varepsilon_{o}$ versus the attenuation $\alpha$ are given in Fig. 9. The curves show that the triangular waveform gives the smallest tracking offset, with the half-sine waveform coming closely behind for all values of $\alpha$, except for the points $\alpha=0$ and $\alpha=1$ where the three cases coincide. The tracking offset depends on the shape of the S-curve and it is changed by the different chip waveforms for early and late sequences.

\section{DISCUSSION}

Improvement in the DLL performance by using half-sine and triangular chip waveforms can be explained with reference to Fig. 2. Under normal operating conditions, the performance of the DLL are determined by the DLL sensitivity (slope of the $\mathrm{S}$-curve at the origin) and the loop noise power. The energies

\section{Maximum Tracking offset $\left(\varepsilon_{0}\right)$}

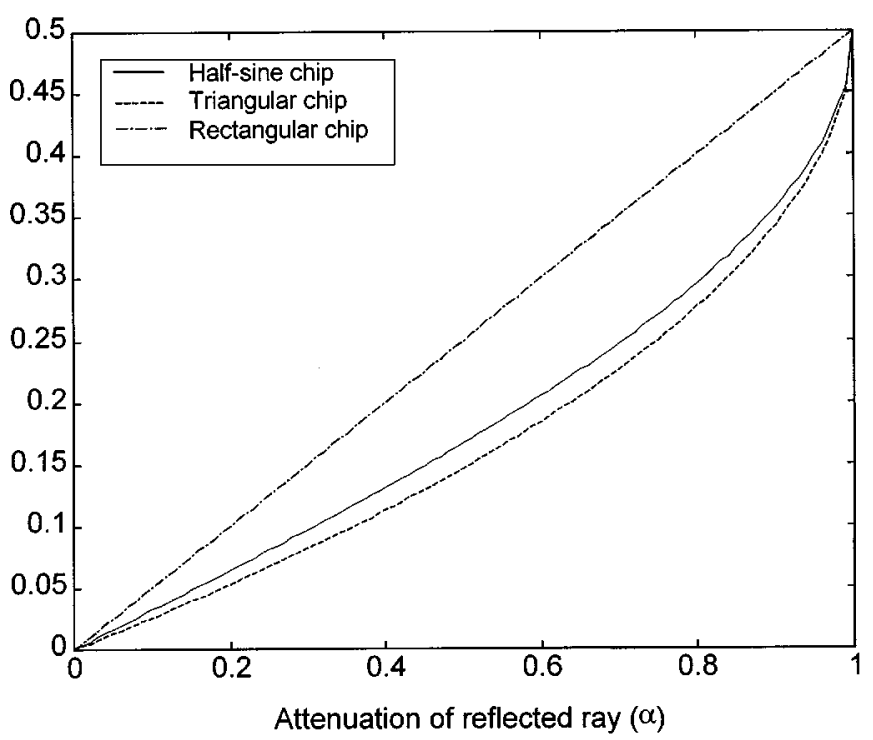

Fig. 9. Plots of tracking offset against reflected ray attenuation.

of the early and late PN chips for both triangular and half-sine waveforms are concentrated around the zero crossings of the incoming PN chip waveform because of the one chip time early-late spacing. This results in only minor effect on the DLL sensitivity compared to that of using rectangular waveform. On the other hand, the loop noise power is uniformly distributed within the chip time when rectangular waveform is used. The half-sine and triangular waveforms reduce the noise power on both sides of the zero crossings and hence reduce the effective loop noise power. Thus, the rms tracking error and the MTLL are, respectively, decreased and increased when the proposed chip waveforms are used. For band-limited signals, the energy distribution is reduced at the zero crossings of the incoming chip. This changes the DLL sensitivity and causes performance degradation.

\section{CONCLUSION}

Two chip waveforms, half-sine and triangular, for locally generated early-late despreading PN signals in a coherent DLL tracking scheme for DS/SS systems have been analyzed for both the ideal and band-limited received signals. It has been shown that the use of either chip waveform reduces the rms tracking error and increases the MTLL considerably when the early-late spacing of the DLL is approximately in the range $0.7-1.3$ chip times. These results apply in particular to the DLL with the commonly used one- chip-time early-late spacing. In addition, it has also been demonstrated that the DLL using triangular or half-sine despreading chip waveforms also reduces the tracking offset in a multipath channel.

\section{REFERENCES}

[1] M. K. Simon, J. K. Omura, R. A. Scholtz, and B. K. Levitt, Spread Spectrum Communications. Rockville, MD: Computer Science, 1985.

[2] S. Haykin, Communication Systems, 3rd ed. New York: Wiley, 1994.

[3] R. L. Peterson, R. E. Ziemer, and D. E. Borth, Introduction to Spread Spectrum Communications. Upper Saddle River, NJ: Prentice-Hall, 1995. 
[4] D. J. R. Van Nee, "Reducing multipath tracking errors in spread-spectrum ranging systems," Electron. Lett., vol. 28, pp. 729-731, Apr. 1992.

[5] — , "Multipath and interference in spread-spectrum tracking systems," in Proc. IEE Colloq. Spread Spectrum Techniques for Radio Communications, London, U.K., 1992, pp. 13/1-13/5.

[6] W. H. Sheen and G. L. Stuber, "Effects of multipath fading on delay locked loops for spread spectrum systems," IEEE Trans. Commun., vol. 42, pp. 1947-1956, Mar. 1994.

[7] R. A. Yost and R. W. Boyd, "A modified PN code tracking loop: Its performance analysis and comparative evaluation," IEEE Trans. Commun., vol. COM-30, pp. 1027-1036, May 1982.

[8] A. Wilde, "Extended tracking range delay-locked loop," in Proc. Intern. Conf. Commun., June 1995, pp. 1051-1054.

[9] - "Delay-locked loop with generalized detector characteristic," Proc. Int. Symp. Spread Spectrum Techniques and Applications, pp. 450-454, Sept. 1996

[10] K. Jamaleddine, D. Vizireanu, F. Malassenet, and S. Halunga, "A new delay locked loop structure with enhanced tracking behavior," in Proc. 7th Int. Symp. Personal, Indoor, and Mobile Communications, Oct. 1996, pp. 1116-1119.

[11] R. Anjaria and R. Wyrwas, "The effect of chip waveform on the performance of CDMA systems in multipath fading noisy channels," in Proc. 42nd IEEE VTS Conf., May 1992, pp. 672-675.

[12] M. A. Landolsi and W. E. Stark, "DS-CDMA chip waveform design for optimal power-bandwidth performance," in Proc. IEEE Int. Symp. Personal, Indoor and Mobile Radio Communications, Sept. 1995, pp. 706-709.

[13] Y. Huang and T. S. Ng, "Performance of coherent receiver with weighted despreading sequence for DS-CDMA," Electron. Lett., vol. 33, pp. 23-25, Jan. 1997

[14] - "A DS-CDMA with power control error using weighted despreading sequences over a multi-path Rayleigh fading channel," IEEE Trans. Veh. Technol., to be published.

[15] — , "A DS-CDMA system using despreading sequences weighted by adjustable chip waveforms," IEEE Trans. Commun., to be published.

[16] — " "Capacity enhancement of band-limited DS-CDMA system using weighted despreading function," IEEE Trans. Commun., to be published.

[17] R. D. Gaudenzi and M. Luise, "Decision-directed coherent delay-lock tracking loop for DS-spread-spectrum signals," IEEE Trans. Commun., vol. 39, pp. 758-765, May 1991.

[18] A. Polydoros and C. L. Weber, "Analysis and optimization of correlative code-tracking loops in spread-spectrum systems," IEEE Trans. Commun., vol. COM-33, pp. 30-43, Jan. 1985.

[19] W. H. Sheen and G. L. Stuber, "A new tracking loop for direct sequence spread spectrum systems on frequency-selective fading channels," IEEE Trans. Commun., vol. 43, pp. 3063-3072, Dec. 1995.

[20] J. K. Holmes and L. Biederman, "Delay-lock-loop mean time to lose lock," IEEE Trans. Commun., vol. COM-26, pp. 1549-1556, Nov. 1978

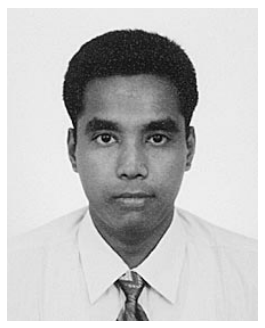

Subramaniam Thayapran was born in Sri Lanka $\mathrm{He}$ received the B.Sc. in electrical and electronic engineering from the University of Peradeniya, Sri Lanka, in 1994. From April 1996 to September 1999, he enrolled in the Ph.D. program with the Department of Electrical and Electronic Engineering, The University of Hong Kong, Hong Kong. His thesis is on the delay-locked loop techniques in DS/SS receivers.

He worked as an Engineer in Sri Lanka Telecom from July 1995 to January 1996 . He is currently an Engineer with Agilent Technologies, Singapore. His research interests include delay-locked tracking and MAI reduction techniques in spread-spectrum systems.

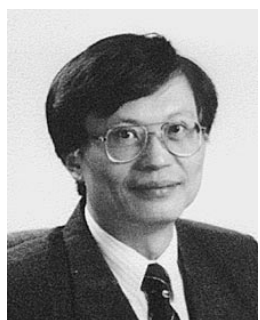

Tung-Sang Ng (S'74-M'78-SM'90) received the B.Sc. (Eng.) with Honors from The University of Hong Kong in 1972, and the M.Eng.Sc. and the Ph.D. degrees from the University of Newcastle, Australia, in 1974 and 1977, all in electrical engineering.

He worked for BHP Steel International and The University of Wollongong, Australia, after graduation for 14 years and returned to Hong Kong in 1991, taking up the position of Professor and Chair of Electronic Engineering at The University of Hong Kong. His current research interests include mobile communication systems, spread spectrum techniques, CDMA, and digital signal processing.

Dr. Ng is a Fellow of the IEE, HKIE, and IEAust. He was the General Chair of ISCAS'97 and is currently VP-Region 10 of IEEE CAS Society. He is also an Executive Committee Member and a Board Member of the IEE Informatics Divisional Board. He has published more than 170 international journal and conference papers. He is currently a Regional Editor of the International Journal-Engineering Applications of Artificial Intelligence (Pergamon Press). He was awarded the Honorary Doctor of Engineering Degree by the University of Newcastle, Australia, in August, 1997 for his service to higher education generally and to engineering education specifically.

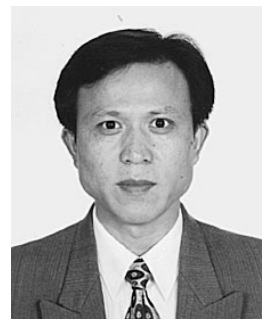

Jiangzhou Wang (M'91-SM'94) received the B.S. and M.S. degrees from Xidian University, Xian, China, in 1983 and 1985, respectively, and the Ph.D. degree (with Greatest Distinction) from the University of Ghent, Belgium, in 1990, all in electrical engineering.

From 1990 to 1992, he was a Postdoctoral Fellow in the University of California at San Diego, La Jolla, where he worked on the research and development of cellular CDMA systems. From 1992 to 1995, he was a Senior System Engineer at Rockwell International Corporation, Newport Beach, CA, where he worked on the development an system design of wireless communications. Since 1995, he has been with the University of Hong Kong, where he is currently an Associate Professor. He is teaching and conducting research in the areas of wireless mobile and spread spectrum communications. He holds a U.S. patent in the GSM system.

Dr. Wang is an Editor for IEEE TRANSACTIONS ON COMMUNICATIONS and a Guest Editor for IEEE Journal on SELECTED AREAS IN COMMUNICATIONS. He has been a Committee Member of a number of international conferences. 